\title{
Full field phase imaging using a harmonically matched diffraction grating pair based homodyne quadrature interferometer
}

\author{
Jigang Wu, ${ }^{\text {a) }}$ Zahid Yaqoob, Xin Heng, Lap Man Lee, Xiquan Cui, and Changhuei Yang \\ Department of Electrical Engineering, California Institute of Technology, Pasadena, \\ California, 91125
}

(Received 31 January 2007; accepted 14 March 2007; published online 13 April 2007)

\begin{abstract}
In this letter, the authors present a novel quadrature interferometry method based on the use of a harmonically matched shallow grating pair. Unlike a simple beam splitter or single shallow grating, the grating pair can confer a nontrivial interference phase shift (other than $0^{\circ}$ or $180^{\circ}$ ) between the output ports of the interferometer. Using the grating pair as the beam splitter/combiner, the authors implement a homodyne quadrature full field phase interferometer and demonstrate the system's capability to acquire phase and amplitude images. (C) 2007 American Institute of Physics.
\end{abstract}

[DOI: $10.1063 / 1.2722685]$

Full field phase based imaging techniques ${ }^{1-4}$ are important for a wide range of applications, such as microscopy and metrology. These methods generally involve interferometry and incorporate some form of nontrivial encoding (in time, space, or polarization) for phase extraction. The encoding process typically entails a more complicated experimental scheme, computationally intensive postprocessing, or some sacrifice in the imaging field of view. In this context, a full field interferometry scheme where the resulting interference outputs are naturally in or close to quadrature can, in principle, simplifies the phase imaging process. However, this requirement is nontrivial. In fact, the outputs of any two-port nonlossy interferometer scheme, including Michelson, Mach-Zehnder, and Sagnac schemes, are constrained to be $180^{\circ}$ shifted (trivial) by energy conservation. We recently demonstrated that a quadrature free-space phase interferometer, termed as the G1G2 interferometer, can be created with a pair of harmonically matched shallow diffraction gratings. ${ }^{5}$ In this letter, we report the following: (1) the creation of the harmonically matched grating pair on a single holographic plate, (2) the use of this single optical element in place of a beam splitter in a modified Mach-Zehnder interferometer and the observation of nontrivial phase between the outputs, and (3) a demonstration of full field phase imaging, which additionally illustrates the utility of phase imaging for flow dynamics studies.

A single shallow diffraction grating can be used to create a multiport $(n \geqslant 3)$ interferometer. However, the outputs of such an interferometer are trivially related in phase. In comparison, the interference between diffractions from the two gratings in a G1G2 interferometer can give rise to nontrivial phase shifts between the outputs. To better explain this concept, we listed the phase of each diffraction order of interest and interference term for a single grating interferometer and a G1G2 interferometer in Fig. 1. In the figure, G1 and G2 are single gratings and their periods $\Lambda_{1}, \Lambda_{2}$ satisfy $\Lambda_{2}=2 \Lambda_{1}, x_{1}$ and $x_{2}$ are the displacements of the single gratings $\mathrm{G} 1$ and G2 with respect to the origin. The phase shift of the $m$ th diffracted order from a shallow grating is given by ${ }^{5}$

\footnotetext{
${ }^{a)}$ Electronic mail: jigang@caltech.edu
}

$$
\phi\left(x_{0}\right)=\left\{\begin{array}{cc}
m\left[\frac{2 \pi x_{0}}{\Lambda}+\frac{\pi}{2}\right], & m \geqslant 1 \\
|m|\left[-\frac{2 \pi x_{0}}{\Lambda}+\frac{\pi}{2}\right], & m \leqslant-1,
\end{array}\right.
$$

where $x_{0}$ is the displacement of the grating from the origin. From Fig. 1(a), we can see that a single grating interferometer can only give rise to a trivial phase shift between the outputs. Although, the phase shift $\phi\left(x_{0}\right)$ is present in the diffracted beams, this $x_{0}$ dependency does not manifest in the interference phase difference between the two outputs. In contrast, the phase difference between the outputs of the G1G2 interferometer depends on the relative displacement of the gratings $\left(x_{1}-x_{2}\right)$ - a quantity that can be adjusted during the G1G2 fabrication process. We note that, while we only use two ports of the G1G2 interferometer, the interferometer is actually a multiport system $(n>=3)$ when higher diffraction orders are considered. It is possible to make use of the other output ports to boost the system sensitivity. We further note that as long as G1 and G2 are exactly harmonically

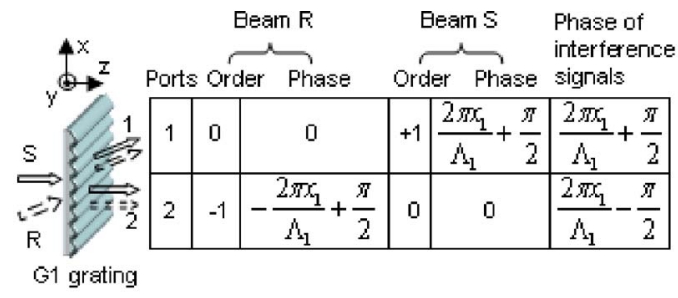

(a)

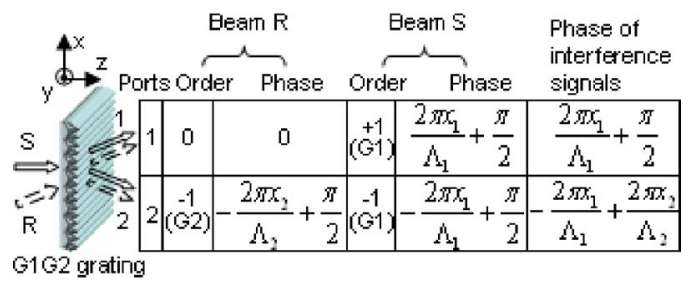

(b)

FIG. 1. Obtaining a nontrivial phase shift in an interferometer by using a harmonically matched grating pair (G1G2 grating) as a beam combiner. (a) The interference outputs of a single grating based interferometer can only possess a $\pi$ phase difference. (b) A G1G2 grating can introduce a nontrivial phase shift between the interference outputs. 


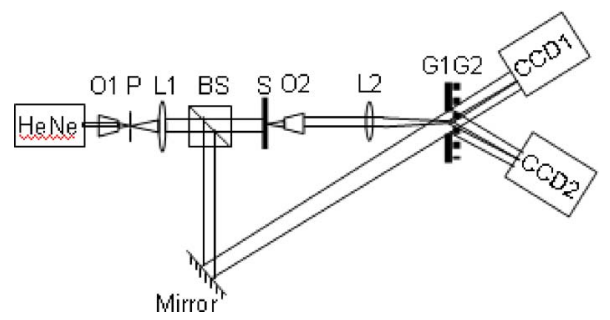

FIG. 2. Mach-Zehnder interferometer setup for phase imaging. $\mathrm{O} 1$ and $\mathrm{O} 2$ : objective lenses 1 and 2; P, pinhole; L1 and L2: lenses 1 and 2; BS: beam splitter; S: sample; G1G2: the harmonically matched grating pair (G1G2 grating) on a holographic plate.

matched, the interference phase difference $\Delta \phi$ will be the same for ray pairs incident at any point on the G1G2 grating - an important consideration for full field phase imaging.

In the regime where $P_{r}$ is much stronger than $P_{s}$, the detected powers at the output ports can be written as

$$
\begin{aligned}
& P_{1} \approx P_{r 1}+2 \sqrt{P_{r 1} P_{s 1}} \cos (\Delta \psi), \\
& P_{2} \approx P_{r 2}+2 \sqrt{\eta} \sqrt{P_{r 2} P_{s 1}} \cos (\Delta \psi+\Delta \phi),
\end{aligned}
$$

where $P_{r 1}$ and $P_{r 2}$ are the detected reference powers at the output ports 1 and 2, respectively; $P_{s 1}$ is the sample power at output port $1 ; \eta=\eta_{-1, G 1} / \eta_{+1, G 1}$, with $\eta_{m, G_{n}}$ denoting the efficiency of the $m$ th diffraction order from grating $G_{n}$ $(n=1,2) ; \Delta \psi=\psi_{s}-\psi_{r}$ is the optical phase difference between the sample and reference beams.

From Eqs. (2) and (3), we get the phase and amplitude of the sample (relative to the reference) beam,

$$
\Delta \psi=\tan ^{-1} \frac{s_{1} \cos \Delta \phi-s_{2}}{s_{1} \sin \Delta \phi}, \quad P_{s 1}=\left(\frac{s_{1}}{\cos (\Delta \psi)}\right)^{2},
$$

where $s_{1}=\left(P_{1}-P_{r 1}\right) / 2 \sqrt{P_{r 1}}$ and $s_{2}=\left(P_{2}-P_{r 2}\right) / 2 \sqrt{\eta P_{r 2}}$. We note that it is still possible to solve for the two unknowns, $P_{s 1}$ and $\Delta \psi$, from the two measurements in the situation where $P_{r}$ is comparable to $P_{s}$. The equations will simply be more complicated than Eq. (4).

Experimentally, we chose to create the G1G2 grating by the following process. An expanded HeNe laser of power of $2.7 \mu \mathrm{W}$ and diameter of $18 \mathrm{~mm}$ was split into three beams. We next arranged the beams to intersect at a standard of 600 lines/mm grating. The angles between the beams were adjusted such that the diffracted orders from different beams overlapped (specifically, we overlapped the $0,+1$, and +2 orders from beams 1,2 , and 3 , respectively, into one spot; the $-1,0,+1$ orders from the same beams into a second spot; and finally the $-2,-1$, and 0 orders into a third spot) and interfered to form bull's eye patterns. The grating was then replaced with a PFG-01 holographic plate. We exposed the plate to the overlapping beams for $\sim 105 \mathrm{~s}$ and processed the resulting plate to create the $\mathrm{G} 1 \mathrm{G} 2$ grating.

The intrinsic interference phase difference of the grating, $\Delta \phi$, was measured by sending a narrow reference and sample beam (diameter of $\sim 1 \mathrm{~mm}$ ) through the plate in the geometry shown in Fig. 2. The uniformity of the phase difference across the plate's surface was verified by repeating the measurement at various points on the plate. Our measurements indicated good uniformity and a $\Delta \phi$ value of $75^{\circ} \pm 10^{\circ}$. $75^{\circ} \pm 10^{\circ}$. the movements of the dye molecules rather than the actual
Downloaded 22 Jun 2007 to 131.215 .225 .9 . Redistribution subject to AlP license or copyright, see http://apl.aip.org/apl/copyright.jsp

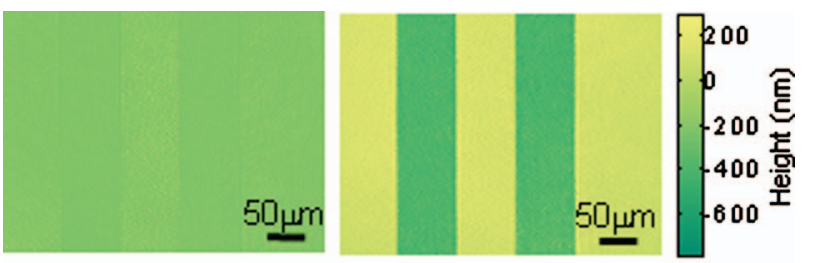

(a)

(b)

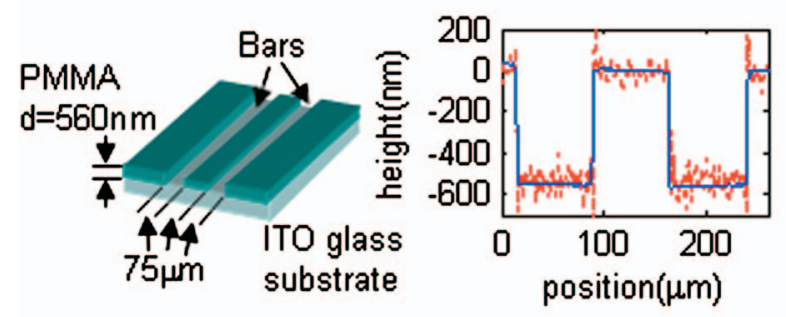

(c)

(d) oxide glass with a thickness of $560 \mathrm{~nm}$. (a) Intensity image; (b) phase image; (c) schematic of the phase object; (d) quantitative phase value compared with the profilometry result. The dotted line (red) and the solid line (blue) are measurements from our phase imaging system and profilometry, respectively.

We next constructed a modified Mach-Zehnder G1G2 interferometer suitable for full field phase imaging (Fig. 2). The laser was focused by objective 1 (Swift 10X), and a pinhole (diameter of $25 \mu \mathrm{m}$ ) served as a spatial filter. The filtered laser beam was then collimated by lens 1 (focal length of $200 \mathrm{~mm}$ ) and split by the $5 / 95$ beam splitter. In the sample arm, objective lens 2 (Newport M-10X) and lens 2 (focal length of $200 \mathrm{~mm}$ ) made up a microscope system that imaged the sample onto two charge coupled device cameras. The harmonic grating pair served as the beam combiner/ splitter. The distance between lens 2 and the G1G2 grating was set at $90 \mathrm{~mm}$. The maximum light intensity incident on the sample was $32.6 \mathrm{~W} / \mathrm{m}^{2}$ and the light intensity of reference beam incident on the grating was $1.7 \mathrm{~W} / \mathrm{m}^{2}$. We measured $\eta_{+1, G 1}=16 \%, \eta_{-1, G 1}=5 \%$, and $\eta_{-1, G 2}=9 \%$; the diffraction efficiencies were determined by the relative power of the light beams during the writing process. During imaging, $P_{1}$ and $P_{2}$ associated with each point on the image plane were measured. Additionally, $P_{r 1}$ and $P_{r 2}$ were measured prior to imaging.

We used this system to image a phase object consisting of two bars of polymethyl methacrylate (PMMA) material (width of $75 \mu \mathrm{m}$, separation of $75 \mu \mathrm{m}$, and height of $\sim 560 \mathrm{~nm}$ ) on a glass plate, as shown in Fig. 3(c) [PMMA's refractive index (ri) is 1.496]. The acquired intensity and phase images are shown in Figs. 3(a) and 3(b). We can see that the intensity image [Fig. 3(a)] shows little contrast. However, the features of the objects are clearly visible in the phase image [Fig. 3(b)]. Quantitative measurement of the PMMA thickness $(\sim 540 \mathrm{~nm})$ is shown in Fig. 3(d); the data agree well with the profilometry measurement $(\sim 555 \mathrm{~nm})$. The standard deviation of the data shown in Fig. 3(d) is $\sim 33 \mathrm{~nm}$, which corresponds to a phase error of $\sim 9.3^{\circ}$.

We next used the phase imaging system to observe liquid diffusion in a microfluidic channel (see Fig. 4). The study of fluidic interfaces in microfluidic channels is important for numerous applications. ${ }^{6}$ Micromixing is usually observed through the use of dyes. These techniques are indirect, as it is
FIG. 3. (Color) Phase image of double bars fabricated on an indium tin 


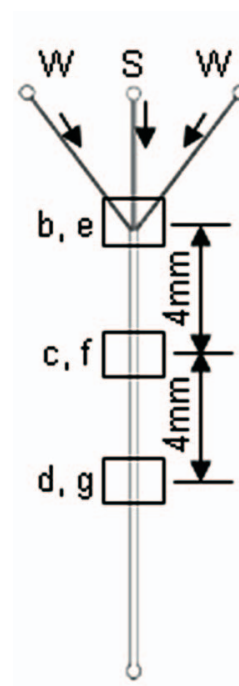

(a)

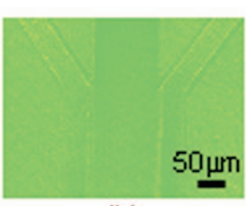

(b)

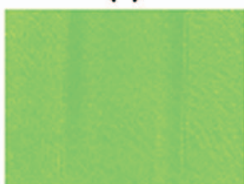

(c)

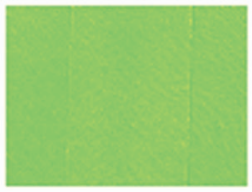

(d)

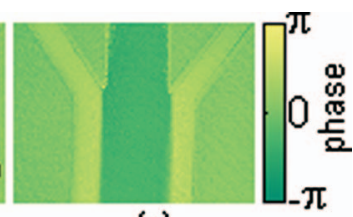

(e)

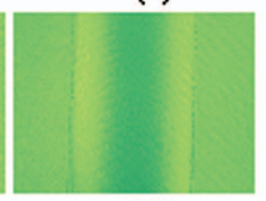

(f)

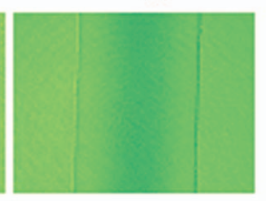

(g)
FIG. 4. (Color) Observing the diffusion process in a microfluidic channel. (a) Microfluidic channel for three fluids mixing. W: water; S: salt water. The location of acquired images is indicated. [(b)-(d)] Intensity images acquired in three different locations as indicated in (a); $[(\mathrm{e})-(\mathrm{g})]$ corresponding phase images of (b)-(d).

fluid molecules that are observed. Phase imaging techniques enable the direct observation of fluid mixing as long as the fluids involved have different ri's. Figure 4(a) shows the fabricated microfluidic channel for observing the mixing of three fluid streams. In the experiment, a $\mathrm{NaCl}$ solution was pumped (weight percentage $=30 \%, \mathrm{ri}=1.393$ ) into the middle inlet of the channel, and two streams of de-ionized water were pumped $(\mathrm{ri}=1.333)$ into the two side inlets. The flow speed was $1 \mathrm{~mm} / \mathrm{min}$ inside the microfluidic channel. Phase [Figs. 4(e)-4(g)] and intensity [Figs. 4(b)-4(d)] images were acquired in the junction region and two downstream locations, as indicated in Fig. 4(a). Once again, the intensity images showed little contrast while the phase images clearly showed the diffusion of the fluids in the channel. This ex- periment demonstrates the utility of our phase imaging technique to study fluid mixing in microfluidic systems.

In summary, we have shown that it is possible to build a full field quadrature interferometer based on a harmonically matched grating pair as the beam splitter/combiner, with which quantitative phase and intensity information associated with a transmissive sample can be measured directly without temporal, spatial, or polarization encoding. As a method, it possesses numerous advantages. First, the implementation and the processing of measurements to extract phase and amplitude information are both relatively simple and easy to implement. Second, the method can, in principle, be adapted for soft $\mathrm{x}$-ray quadrature phase imaging. While a wide range of optical elements does not have effective soft $\mathrm{x}$-ray regime equivalents and as such a range of optical techniques cannot be translated for $\mathrm{x}$-ray applications, an $\mathrm{x}$-ray regime version of the diffraction grating pair can be manufactured with current fabrication technology. Third, unlike a conventional beam splitter, a harmonic grating pair is flat, thus it can be directly fabricated into a layered microstructure with ease. An on-chip quadrature phase imaging system has the potential to be very useful as an underlying base of a microfluidic device for providing a convenient and direct means to monitoring flow dynamics in microfluidic flows.

The authors would like to acknowledge Emily McDowell for helpful discussion and Liang Feng from UC San Diego for help in fabrication. This project is supported by NSF BES-0547657.

${ }^{1}$ K. Creath, Prog. Opt. 26, 349 (1998).

${ }^{2}$ P. Marquet, B. Rappaz, P. J. Magistretti, E. Cuche, Y. Emery, T. Colomb, and C. Depeursinge, Opt. Lett. 30, 468 (2005).

${ }^{3}$ T. Ikeda, G. Popescu, R. R. Dasari, and M. S. Feld, Opt. Lett. 30, 1165 (2005).

${ }^{4}$ D. O. Hogenboom, C. A. DiMarzio, T. J. Gaudette, A. J. Devaney, and S. C. Lindberg, Opt. Lett. 23, 783 (1998).

${ }^{5}$ Z. Yaqoob, J. Wu, X. Cui, X. Heng, and C. Yang, Opt. Express 14, 8127 (2006).

${ }^{6}$ J. Atencia and D. J. Beebe, Nature (London) 437, 648 (2005). 